\title{
Finalidades de la enseñanza de la historia en futuros profesores
}

Purposes of the Teaching of History in Future Teachers

Artículo de investigación | Research Article

Fecha de recepción: 14 de diciembre de 2017

Fecha de aceptación: 8 de febrero de 2019

Fecha de disponibilidad en línea: marzo de 2020

doi: 10.11144/Javeriana.m13.fehf

Gustavo Alonso González-Valencia gustavo.gonzalez@uab.cat

Universidad Autónoma de Barcelona, España

ORCID: https://orcid.org/0000-0001-8718-417X

Antoni Santisteban-Fernández

Antoni.santisteban@uab.cat

Universidad Autónoma de Barcelona, España

(D) ORCID: https://orcid.org/0000-0001-7978-5186

Joan Pagès-Blanch

Joan.Pages@uab.cat

Universidad Autónoma de Barcelona, España

ORCID: https://orcid.org/0000-0001-8650-3976

Para citar este artículo | To cite this article

González-Valencia, G. A.; Santisteban-Fernández, A. \& Pagès-Blanch, J. (2020).

Finalidades de la enseñanza de la historia en futuros profesores. magis, Revista

Internacional de Investigación en Educación, 13, 1-23. doi: 10.11144/Javeriana.

m13.fehf 


\title{
Resumen
}

Este artículo presenta los resultados de una investigación que indagó acerca de las representaciones sociales que sobre las finalidades de la enseñanza de la historia tiene un grupo de futuros profesores de historia. Los participantes cursaban el último año de estudios, período en el que realizan las prácticas de fin de carrera. La investigación fue mixta, tanto en la recolección como en el análisis de la información. Los resultados muestran que las representaciones son heterogéneas, están influenciadas fundamentalmente por la formación disciplinar y en menor medida por las prácticas.

\section{Palabras clave}

Formación de docentes; enseñanza de la historia; práctica pedagógica; historia

\begin{abstract}
This article shows the results from a research that delved into the social representations by a group of future history teachers regarding the purposes of teaching history. The participants were in the last year of the study program, and they had to do the teaching practice in order to graduate. This research uses a combined methodology both in the data gathering and in the information analysis. The results indicate that the representations are heterogeneous, influenced basically by the disciplinary formation and, to a lesser extent, by the practices.
\end{abstract}

\section{Keywords}

Teacher education; history education; teaching practice; history 
Descripción del artículo | Article description

El artículo presenta los resultados de una investigación que

indagó acerca de las finalidades de las ciencias sociales y

la enseñanza de la historia en particular, en el marco del

proyecto Representaciones acerca de la enseñanza de las

ciencias sociales.

El estudio fue realizado en el marco del Grupo de

Investigación en Didáctica de las Ciencias Sociales de la

Universidad Autónoma de Barcelona, UAB.

\section{Introducción}

La formación inicial del profesorado es una actividad planificada con la mayor coherencia posible. Es usual leer que su finalidad es preparar el profesorado con el conocimiento pedagógico, didáctico y disciplinar suficiente para diseñar, desarrollar y evaluar procesos de enseñanza y de aprendizaje, reflexionar sobre sus prácticas y proponer innovaciones (González-Valencia, 2013; Pagès-Blanch, 2004). No obstante, los futuros profesores perciben una brecha significativa entre lo enunciado en los discursos presentes en la formación y lo que acontece en las prácticas (González-Valencia, 2013; Harris, 2012). Por lo anterior, identificar y comprender cómo los estudiantes de último año de formación representan la enseñanza de la historia motivó el estudio que da origen a este artículo.

La investigación acerca de las representaciones sociales (RS) que posee el profesorado sobre la enseñanza de la historia, la geografía y las ciencias sociales es una línea de investigación consolidada en la Didáctica de las Ciencias Sociales (DCS) (González-Valencia \& Santisteban-Fernández, 2014; Henríquez \& Pagès-Blanch, 2004; Pagès-Blanch, 2002b; Pagès-Blanch \& Santisteban-Fernández, 2014; Prats, 1999). En las últimas décadas, esta ha tenido un número significativo de trabajos que evidencian sus desarrollos (Adler \& Goodman, 1983; Evans, 1991; Harris, 2012; Hernández-Cervantes, 2014; Jara, 2012; Pagès-Blanch, 1999; Pantoja-Suárez, Loaiza-Zuluaga \& Posada-López, 2014; Reitano \& Green, 2013; Santisteban-Fernández, 2010; Valencia-Castañeda, 2014; Valencia-Castañeda, Pérez \& Jara, 2014; Villalón-Gálvez, 2012, 2015; Voet \& De Wever, 2016; Yilmaz, 2008). Los participantes de la mayoría de los estudios fueron estudiantes en su período de formación teórica o profesionales en ejercicio; sin embargo, son escasos los trabajos con profesores de historia en formación, que realizan sus prácticas de último año. Uno de los pocos trabajos en esta dirección es el de Miguel Jara, Víctor Salto y Fabiana Értola (2016), quienes indagaron por la forma en que los estudiantes representan la enseñanza de la historia. 
En este trabajo, los autores hallaron una desconexión entre lo que se puede denominar como enseñanza, en general, y los planteamientos propios de la Historia.

El período de prácticas de fin de carrera es una fase relevante de la formación del futuro profesor de historia, porque representa un cambio de roles y de escenarios. El primero se concreta en el paso de estudiante a profesor y el segundo es la transición de la universidad a la institución educativa donde realizará su labor profesional (Davini, 2002). Con las prácticas se espera que los estudiantes le encuentren sentido a la formación que recibieron en la universidad en la medida en que ejerzan como profesores, asuman su rol profesional y conozcan el funcionamiento de las instituciones educativas, entre otros.

En este marco de referencia, la investigación se preguntó: ¿Cómo el profesorado en formación representa las finalidades de la enseñanza de la historia? Así pues, se pretendió desvelar y comprender el valor educativo que los participantes del estudio le confieren a esta asignatura (Harris, 2012; Pagès-Blanch, 2002a; Wineburg, 2001).

El trabajo asumió que las RS son un tipo de conocimiento que es construido a partir de:

nuestras experiencias, pero también de las informaciones, conocimientos y modelos de pensamiento que recibimos y transmitimos a través de la tradición, la educación y la comunicación social. De este modo, ese conocimiento es, en muchos aspectos, un conocimiento socialmente elaborado y compartido (Denise Jodelet, en Moscovici, 1984, p. 473).

Esta perspectiva permite considerar la influencia de otros escenarios y dimensiones, sin reducirla a la formación reglada y al aspecto cognitivo. Develar lo que el profesorado piensa bajo el planteamiento de las RS, está en la línea de evidenciar el trasfondo ideológico de las decisiones y acciones de las personas (Phoenix, Howarth \& Philogène, 2017). Indagar las RS es un interés que se enmarca en la Teoría Crítica (Mora, 2002), porque se asume que "detrás de cada teoría y práctica educativa hay siempre unos valores sociales, aunque no se formulen de manera concreta o sistemática" (Gimeno, 1999, p. 14). Estos valores están presentes en todo momento y se concretan en qué se enseña del pasado y cómo se hace (Kitson, Steward \& Husbands, 2015).

La teoría de las RS ha sido empleada en la investigación en DCS; tal es el caso de Miguel Jara, Víctor Salto y Fabiana Értola (2016), quienes plantean que estas se pueden entender como "formas de pensar y organizar la realidad social, constituidas por elementos de carácter simbólico: imágenes, creencias, actitudes, opiniones y estereotipos" (p. 7). En el estudio de las RS 
hay diferentes aproximaciones teóricas y metodológicas. Esta investigación asumió la Teoría del Núcleo Central, la cual plantea que, en el proceso de construcción de las RS, la estructura y organización son esenciales para identificarlas y caracterizarlas (Rodríguez-Salazar, 2007).

Al estudiar las RS acerca de las finalidades de la historia, se pretende develar los marcos que emplean los futuros profesores cuando piensan su enseñanza. Con el conocimiento construido se pretende contribuir a cualificar los procesos de formación inicial y permanente del profesorado a través de innovaciones que lleven a "conseguir un cambio conceptual [y en las RS] y evitar que dichas representaciones actúen como obstáculos para su formación didáctica" (Pagès-Blanch, 1999, p. 167) o, tal como lo plantean Miguel Jara, Víctor Salto y Fabiana Értola (2016), que su finalidad sea tomar conciencia, dar sentido y transformar, en este caso, la enseñanza de la historia.

\section{Las finalidades de la enseñanza de la historia}

Cuando se diseña un proceso de enseñanza y aprendizaje, una de las preguntas centrales que se intenta responder es ipara qué se enseña determinada ciencia o disciplina? (Gómez-Carrasco, Ortuño-Molina \& MolinaPuche, 2014; González-Valencia, 2013; Harris, 2012; Jara, Salto \& Értola, 2016; Wineburg, 2001). En el caso de la historia, las respuestas se abordan desde diferentes perspectivas como son: para que los estudiantes aprendan los hechos más importantes de la historia, para que conozcan su territorio y su cultura, para que sean buenos ciudadanos, para que comprendan el legado cultural, etc. La lista puede ser tan extensa como el profesorado en el que se indague. Las respuestas también tienden a generar un amplio debate social, político y académico (Voet \& De Wever, 2016). Este trabajo asumió que la finalidad de la enseñanza de la historia es:

... contrasocializadora, es decir, ha de preparar al alumnado para que construya sus propios conocimientos, se ubique en su mundo y esté preparado para intervenir en él de manera democrática. Se reclama educar la coherencia entre el pensamiento y la acción a fin de garantizar que el protagonismo de los jóvenes ciudadanos y ciudadanas en la construcción del mundo personal y social se realice desde valores alternativos basados en el compromiso, la justicia, la tolerancia y la solidaridad (Pagès-Blanch, 2002a, p. 258).

Lo anterior puede ser complementado con la idea de llevar a los estudiantes a construir pensamiento histórico, social, crítico y creativo 
(Benejam-Argimbau, 2002; Dewey, 1978; Rodríguez-Ávila, 2014; Ross, 2006; Santisteban-Fernández, 2009; Singer, 2008; Virta, 2002). Por lo tanto, la finalidad de la enseñanza de la historia no puede limitarse al aprendizaje de conceptos (Wineburg, 2001) y su repetición en las clases, porque "Los conocimientos, las habilidades y las actitudes [asumen] sentido cuando los [estudiantes los] utilizan en la comprensión de los problemas de la vida y en su resolución, es decir, cuando implican reflexión y acción" (Pagès-Blanch, 2009, p. 10). En palabras de Diana Pipkin (2009), los aprendizajes son relevantes cuando son empleados para comprender y actuar en la realidad. En esta perspectiva, se entiende que "los niños y las niñas, los chicos y las chicas pueden, y deben, aprender historia. Han de aprender historia para poder actuar como ciudadanos y ciudadanas de una sociedad que apuesta por la democracia como forma de convivencia" (Pagès-Blanch, 2007, p. 13).

A partir de lo anterior, resulta necesario que el profesorado en formación piense y reflexione acerca de las finalidades de la enseñanza de la historia (Gómez-Esteban, 2004; Harris, 2012; Jara, Salto \& Értola, 2016; Pluckrose, 1996; Wineburg, 2001) y de qué manera contribuyen al desarrollo del pensamiento histórico y social. Por lo tanto, es relevante que el profesorado conozca sus fundamentos teóricos, concretados en conceptos (de primer y segundo orden), los cuales se convierten en coordenadas conceptuales y metodológicas con las cuales explicar y comprender los hechos del pasado, el presente y pensar el futuro (Harris, 2012; PagèsBlanch \& Santisteban-Fernández, 2014; Santisteban-Fernández, 2017; Wineburg, 2001).

\section{La formación del profesorado para enseñar historia}

La formación del profesorado para enseñar historia en Colombia la realizan, fundamentalmente, las universidades a través de programas denominados licenciaturas en educación básica con énfasis en ciencias sociales. La normativa define que los programas en "Educación corresponden a un campo de acción cuya disciplina fundante es la pedagogía, incluyendo en ella la didáctica, por cuanto constituye un ámbito de reflexión a partir del cual se genera conocimiento propio que se articula interdisciplinariamente" (Colombia, MEN, 2004, p. 2).

El componente disciplinar, en este caso la historia, está relacionado con los discursos de la pedagogía, la didáctica general y la didáctica de la disciplina, en este caso, de la historia y las ciencias sociales. Este tipo de programas suele tener una duración de 5 años. 
En términos teóricos, la formación inicial del profesorado es una fase en la que se espera desarrollar en los futuros docentes un conjunto de conocimientos y experiencias que les permita desempeñarse de manera competente en el diseño, materialización y evaluación de prácticas de enseñanza (González-Valencia, 2013; Liston \& Zeichner, 2003). Los conocimientos específicos se centran en los fundamentos de la historia, la geografía y las ciencias sociales y las didácticas específicas. Algunos estudios señalan que en los programas de formación inicial del profesorado se suele hacer énfasis en el conocimiento de los fundamentos teóricos y metodológicos de las ciencias de referencia, en detrimento de una formación didáctica para su enseñanza (Coudannes-Aguirre, 2010; Sánchez-Agustí, 2011). Michiel Voet y Bram de Wever (2016) consideran que en las universidades los futuros profesores tienden a conocer los fundamentos de la historia en profundidad, antes que sus dimensiones educativas. Esto puede ser problemático por la naturaleza del desempeño como profesores de historia, lo que requiere el conocimiento y la competencia en las dos dimensiones.

\section{El diseño de la investigación}

La investigación realizada fue mixta (Hernández-Sampieri, FernándezCollado \& Baptista-Lucio, 2014; Teddlie \& Tashakkori, 2009). Las técnicas de recolección utilizadas fueron el cuestionario, la entrevista y el grupo de discusión. El cuestionario inicial $(n=50)$ tuvo como finalidad construir la primera aproximación a la estructura de las representaciones. El cuestionario final permitió hacer una comparación entre dos momentos de la investigación. La entrevista $(n=19)$ buscó comprender con mayor detalle la estructura y el contenido de las representaciones. El grupo focal $(n=4)$ fue una técnica empleada para obtener información que permitiera configurar una triangulación. La implementación de las técnicas se hizo en este mismo orden mencionado. Los instrumentos fueron construidos a partir de la revisión de la literatura y sometidos a un juicio de expertos $(n=3)$; se hizo una prueba piloto con 20 estudiantes del curso anterior al de los participantes. La combinación de estas amplió la posibilidad de conocer en profundidad lo que las personas piensan sobre un hecho o categoría conceptual. El análisis de los datos cuantitativos se realizó a través de estadística descriptiva, para esto se empleó el paquete estadístico SPSS. Para los datos cualitativos se usó el análisis de contenido (Bardin, 1986; Strauss \& Corbin, 2002) y del discurso (Van Dijk, 1992; Íñiguez-Rueda, 2006; Ruiz, 2008), el cual se concretó a través de la codificación teórica (Flick, 2007). En esta fase se hizo uso del software Atlas.ti. 
La investigación contó con la participación de 50 estudiantes de una titulación para profesor de Ciencias Sociales que, a la fecha, era la totalidad de los que cursaban el último año de su formación. En este período, los estudiantes adelantaban las prácticas finales en instituciones de enseñanza obligatoria, en la que realizaban docencia directa de temas relativos a la historia, la geografía y las ciencias sociales por 10 horas a la semana durante 30 a 32 semanas. Se eligió esta fase de la formación porque se consideró que la experiencia de la práctica podría tener algún tipo de influencia en la configuración de las representaciones. La información se obtuvo a lo largo del período académico. Los primeros datos se recogieron al inicio del semestre a través de un cuestionario. El análisis inicial permitió identificar informantes clave para realizar las entrevistas ( $n=19$ ). Para esta actividad, las personas fueron seleccionadas siguiendo criterios de edad, género y perfil de la estructura de la representación identificada en el análisis estadístico. Durante este tiempo, los participantes hicieron parte de un espacio de formación denominado Seminario Permanente de Práctica (SPP), en el cual se propuso una reflexión acerca de la experiencia de enseñar historia. En esta actividad, se obtuvo información pertinente para la investigación a través de un taller sobre las finalidades de la enseñanza de la historia, el cual fue contestado en grupos de discusión $(n=4)$. Al finalizar el período de estudios, los estudiantes respondieron el cuestionario por segunda vez.

El diseño del cuestionario - de manera puntual la pregunta por las finalidades de la enseñanza de la historia - tomó como referencia la propuesta de Jürgen Habermas (1966) sobre los intereses que orientan la generación de conocimiento: técnico, práctico y emancipatorio. Esta propuesta se ha empleado en otros trabajos en la DCS, la historia y la geografía (Benejam-Argimbau, 2002; Catling, 2004; Evans, 1991; González-Valencia \& Santisteban-Fernández, 2016).

\section{Resultados}

\section{Las finalidades de la historia en el cuestionario inicial}

El cuestionario tuvo una pregunta relativa a las finalidades de la enseñanza de la historia en la educación obligatoria. Esta pregunta estaba integrada por doce ítems. Los participantes debían elegir los tres ítems que consideraban más relevantes. El análisis estadístico arrojó los siguientes resultados (Tabla 1). 
Tabla 1

Clasificación de las finalidades de la enseñanza de la Historia. Cuestionario inicial ${ }^{1}$

\begin{tabular}{l|c}
\hline $\begin{array}{l}\text { Finalidades de enseñanza de la historia } \\
\text { (cuestionario inicial) }\end{array}$ & $\begin{array}{c}\text { Porcentaje } \\
\text { (\%) }\end{array}$ \\
\hline Comprender el presente a partir del pasado & 28,0 \\
\hline Comprender el cambio y la continuidad en las sociedades & 22,0 \\
\hline Desarrollar el espíritu crítico & 17,3 \\
\hline Pensar el futuro a partir de las referencias del pasado & 10,7 \\
\hline Reconocer los hechos más relevantes de la historia de la humanidad & 7,3 \\
\hline Construir una identidad colectiva & 4,7 \\
\hline Conocer la historia nacional & 3,3 \\
\hline Construir saberes a partir de la lectura de documentos & 2,7 \\
\hline Conocer las diferentes épocas históricas & 2,0 \\
\hline Reconocer el patrimonio & 1,3 \\
\hline Establecer una cronología de hechos & 0,7 \\
\hline Situar los personajes importantes y sus acciones relevantes ${ }^{2}$ & 0,0 \\
\hline Total & 100,0 \\
\hline
\end{tabular}

Fuente: elaboración propia (SPSS)

La anterior clasificación es sugerente por sí misma, pero cobra mayor sentido cuando se analiza su distribución siguiendo la propuesta de Jürgen Habermas (1966). La perspectiva con mayor peso es la emancipatoria con $78 \%$. La segunda corresponde a la práctica con 16\% y, la tercera, a la técnica con 6\% (Tabla 2).

Tabla 2

Distribución de finalidades por intereses en historia

\begin{tabular}{|c|c|c|}
\hline Interés & (\%) & Finalidades \\
\hline Emancipatoria & 78 & $\begin{array}{l}\text { - Comprender el presente a partir del pasado } \\
\text { - Comprender el cambio y la continuidad en las sociedades } \\
\text { - Desarrollar el espíritu crítico } \\
\text { - Pensar el futuro a partir de las referencias del pasado }\end{array}$ \\
\hline Práctica & 16 & $\begin{array}{l}\text { - Reconocer los hechos más relevantes de la historia de } \\
\text { la humanidad } \\
\text { - Construir una identidad colectiva } \\
\text { - Construir saberes a partir de la lectura de documentos } \\
\text { - Reconocer el patrimonio }\end{array}$ \\
\hline Técnica & 6 & $\begin{array}{l}\text { - Conocer la historia nacional } \\
\text { - Conocer las diferentes épocas históricas } \\
\text { - Establecer una cronología de hechos y situar los personajes } \\
\text { importantes y sus acciones relevantes }\end{array}$ \\
\hline
\end{tabular}

Fuente: elaboración propia

1 La tabla es el resultado del análisis de tendencias con respuesta múltiple realizado con SPSS.

2 Este último ítem no fue elegido por ninguna persona, mostrando una tendencia en las preferencias, lo cual es importante para una investigación didáctica. 
La distribución de las finalidades puede deberse a que los estudiantes, durante su formación en historia, tuvieron profesores que la asumían desde tres perspectivas: una hacía énfasis en los acontecimientos o en la historia evenemencial o factual; en otra, la historia se asumió como la comprensión del pasado y, la última, tuvo una fuerte connotación social y crítica que se asocia a una perspectiva marxista. Esta diversidad puede explicar la distribución de las finalidades, siendo respaldada por comentarios como el siguiente, que se realizaron durante las entrevistas:

Es que en Historia hemos tenido por lo menos dos clases de profesores: a unos que lo que les interesa es que uno recuerde la Historia clásica, con datos y fechas, pero también tenemos a otros con la perspectiva de los Annales o el marxismo, entonces ahí uno puede ver como dos ideas diferentes de la Historia. Cuando uno escucha a uno u otro profesor, pues uno ya se identifica ¿cierto? (E19).

\section{Las finalidades de la historia en las entrevistas}

En el análisis cualitativo, la propuesta de Jürgen Habermas mostró ser poco útil porque era un corsé que restringía y dejaba por fuera una buena parte de la información. El análisis mostró la existencia de matices en el conjunto de los datos; por lo tanto, se decidió que esta era asumida como un punto de referencia en esta parte del análisis. A partir de lo anterior, la alternativa metodológica fue construir tipologías, las cuales fueron emergiendo en el proceso. Estas cumplieron dos propósitos: dar cuenta de los matices y ampliar las posibilidades de comprensión de los datos.

En una de las sesiones del SPP, se preguntó a los participantes: ¿para qué y por qué los estudiantes deben aprender historia y ciencias sociales? Esta fue contestada en grupos de 4 a 6 personas. La información permitió ampliar la comprensión de las RS sobre las finalidades de la enseñanza de la historia. Además, fue prefigurando el núcleo central de las RS a partir de la identificación de 5 tipologías que se presentan en la Tabla 3 y se caracterizan a continuación.

Tabla 3

Tipologías acerca de las finalidades de la enseñanza de la historia

\section{Tipología}

Tipología No. 1. La historia factual, de la nación y la patria

Tipología No. 2. La historia factual y el conocimiento de la realidad objetiva

Tipología No. 3. La historia como conocimiento de la diversidad y la convivencia

Tipología No. 4. La historia social y la comprensión de la realidad

Tipología No. 5. La historia social y política para la transformación social

Fuente: elaboración propia 
Tipología No. 1. La historia factual, de la nación y la patria

Esta se caracteriza por considerar que en la enseñanza y el aprendizaje de la historia es fundamental conocer los hechos relevantes, con sus personajes, lugares y fechas, así como las tradiciones. Lo anterior permite conocer cómo funciona el mundo. El pasado se presenta como una verdad absoluta. Esta tipología aparece como la minoritaria y se refleja en comentarios como los siguientes:

Por ejemplo, para mí es muy importante que los estudiantes reconozcan cosas relevantes de nuestra historia, como lugares, fechas y todo eso, ime entiende? (E19).

Porque deben saber cómo está conformado el mundo y cómo es su funcionalidad (ASG1).

Tipología No. 2. La historia factual y el conocimiento de la realidad objetiva

Está centrada en el reconocimiento de la tradición, el pasado y el aprendizaje de hechos relevantes, lo cual se asume como esencial para aprender a vivir en sociedad. En esta tipología se ubican los participantes que intentan distanciarse de una idea de la historia centrada en las categorías de nación y patria; esto plantea que el conocimiento objetivo de los hechos históricos es suficiente para que los estudiantes aprendan a valorar su realidad cercana (la región), para así configurar su identidad personal, regional y nacional. En esta tipología aparecen matices que sugieren la transición entre la perspectiva técnica y práctica de Jürgen Habermas. Los siguientes comentarios reflejan este grupo:

¿Qué les interesa? —refiriéndose a sus estudiantes—. Por ejemplo, ahora estábamos hablando de la historia; la historia regional de ellos se les hace más interesante. ¿Por qué? Porque fue un proceso que está, que pasó acá, para poder fundar esta ciudad. ¿Sí ve? Entonces es importante que ellos lo sepan porque ellos están viviendo acá. Ellos deben saber del espacio geográfico que utilizan, que el acontecer histórico que ha venido pasando para que nosotros estemos acá (E32).

Para conocer los procesos históricos contextualizándolos a su realidad, para situarse en el mundo actual y así crear una identidad (ASG6).

Tipología No. 3. La bistoria como conocimiento y para la convivencia.

Este grupo de participantes asocia que, al aprender historia, los estudiantes podrán mejorar el conocimiento histórico y entender las sociedades, de dónde vienen y hacia dónde van. Estos planteamientos sugieren un interés por conocer y comprender algunos conceptos (de primer y segundo orden) con el fin de tener coordenadas que les permitan ubicarse en el 
mundo en el que conviven con otros. Los participantes también reconocen que la historia es dinámica y construida por las personas. Esta tipología se aprecia en los siguientes comentarios:

Para comprender su realidad, conociendo su historia para relacionarla con su presente (ASG7).

Creo o considero que el ser humano nació para vivir en sociedad y, como tal, los jóvenes deben conocer cómo ha sido la evolución del ser humano en su entorno social, cuál ha sido su historia, cómo ha sido su comportamiento y su expansión por el mundo (ASG4).

Tipología No. 4. La historia socialy la comprensión de la realidad

Este grupo plantea que la enseñanza de la historia debe estar orientada a que los estudiantes conozcan y comprendan el contexto social cercano; por lo tanto, la asumen como fundamental para la convivencia. En las respuestas mencionan que la enseñanza debe llevar a la comprensión de los hechos históricos (interpretación histórica). Se puede señalar que se trasciende lo factual y lineal (de manera concreta, emergen conceptos como cambio y continuidad, explicación causal). En esta tipología, se hace explícito el rol de las personas en la construcción de los procesos sociales, se asume que la historia es próxima y se sugiere que su enseñanza tiene un horizonte político. Así lo muestran los siguientes comentarios:

Yo pienso que por eso son importantes las ciencias sociales, porque ayudan al estudiante, primero a enfocarse que está en un lugar determinado y que alrededor suyo pasan muchos acontecimientos y que tiene que conocerlos, porque si no los conoce realmente no puede afrontar muchas situaciones que se le presenten, porque no tienen los argumentos, no saben analizar, no saben discernir, porque no tienen las herramientas necesarias para enfrentar diferentes problemas que se le presenten (E25). Realizar un análisis y comprender que los hechos del pasado tienen unas consecuencias en nuestro presente, busca dar respuesta a la relación hombre-medio con toda la interacción que compete en el ámbito social, económico, político, etc. (ASG9).

\section{Tipología No. 5. La historia social y politica para la transformación social}

En esta, el profesorado en formación hace explícito el interés por ir más allá de lo evidente en los hechos históricos, y emergen otras categorías conceptuales asociadas a otras ciencias sociales y humanas (poder, estructura social, cultura, sistema económico, territorio), las cuales contribuyen a la interpretación y comprensión de los hechos. En algunas respuestas, los participantes elaboran explicaciones causales para respaldar sus 
comentarios. Este grupo asume que la historia la construyen las personas y que la explicación, interpretación y comprensión histórica son necesarias para la transformación social. Esta tipología es la de mayor número de participantes y se refleja en comentarios como los siguientes:

Para mí es fundamental eso: entender como el proceso que ha tenido el hombre a través del tiempo para llegar a lo que es ahora y para entender la realidad social que está viviendo (E2).

Para entender y comprender los cambios que se dan en su entorno social, en su país. Esos cambios pueden ser físicos, sociales, económicos y ambientales. Es importante que asimilen dichos cambios, para que no choquen con el proceso, que conozcan para que protejan y defiendan sus derechos, para que participen en las conversaciones a nivel familiar y local, si es el caso, y para que contribuyan en la formación de las generaciones siguientes (ASG10).

\section{Las finalidades de la historia en el cuestionario final}

Al finalizar el SPP y el período académico, los participantes contestaron el cuestionario por segunda vez. A partir de las respuestas, se configuró la siguiente clasificación.

Tabla 4

Clasificación de las finalidades de la enseñanza de la historia. Cuestionario final${ }^{3}$

\begin{tabular}{l|c}
\hline $\begin{array}{l}\text { Finalidades de la historia } \\
\text { (cuestionario final) }\end{array}$ & $\begin{array}{c}\text { Porcentaje } \\
\mathbf{( \% )}\end{array}$ \\
\hline Comprender el presente a partir del pasado & 23,6 \\
\hline Comprender el cambio y la continuidad en las sociedades & 21,8 \\
\hline Desarrollar el espíritu crítico & 16,4 \\
\hline Pensar el futuro a partir de las referencias del pasado & 16,4 \\
\hline Construir una identidad colectiva & 7,3 \\
\hline Establecer una cronología de hechos & 5,5 \\
\hline Reconocer los hechos más relevantes de la historia de la humanidad & 5,5 \\
\hline Construir saberes a partir de la lectura de documentos & 1,8 \\
\hline Conocer las diferentes épocas históricas & 1,8 \\
\hline Reconocer el patrimonio & 0,0 \\
\hline Conocer la historia nacional & 0,0 \\
\hline Situar los personajes importantes y sus acciones relevantes & 0,0 \\
\hline Total & 100,0 \\
\hline
\end{tabular}

Fuente: elaboración propia (SPSS)

3 Esta tabla es el resultado de un análisis de los valores relativos con respuestas múltiples. 
La comparación de ambos cuestionarios evidencia dos tipos de cambios: el primero en la reducción del número de finalidades elegidas y, el segundo, en la modificación del peso relativo de algunas. El número de finalidades pasó de 11 en el cuestionario inicial a 9 en el final. En esta clasificación desaparecieron las siguientes opciones: Conocer la historia nacional, Reconocer el patrimonio y Situar los personajes importantes y sus acciones relevantes. En el valor relativo de las perspectivas, hay cambios que se pueden valorar como no significativos en términos estadísticos. Los datos muestran que el grupo de finalidades correspondiente a la perspectiva emancipatoria se mantuvo en $78 \%$. La perspectiva práctica se redujo en 1,4\%, es decir, pasó de 16\% a 14,8\%. La instrumental disminuyó el número de finalidades e incrementó su peso relativo, en el cuestionario inicial fue del $6 \%$ y en el final de $7,2 \%$.

Tabla 5

Distribución de finalidades por intereses en historia

\begin{tabular}{|c|c|c|}
\hline Interés & $(\%)$ & Finalidades \\
\hline Emancipatoria & 78,0 & $\begin{array}{l}\text { - Comprender el presente a partir del pasado } \\
\text { - Comprender el cambio y la continuidad en las sociedades } \\
\text { - Desarrollar el espíritu crítico } \\
\text { - Pensar el futuro a partir de las referencias del pasado }\end{array}$ \\
\hline Práctica & 14,8 & $\begin{array}{l}\text { - Reconocer los hechos más relevantes de la historia de } \\
\text { la humanidad } \\
\text { - Construir una identidad colectiva } \\
\text { - Construir saberes a partir de la lectura de documentos }\end{array}$ \\
\hline Técnica & 7,2 & $\begin{array}{l}\text { - Conocer las diferentes épocas históricas } \\
\text { - Establecer una cronología de hechos y situar los personajes } \\
\text { importantes y sus acciones relevantes }\end{array}$ \\
\hline
\end{tabular}

Fuente: elaboración propia

A partir de estos datos, se puede decir que el grupo de futuros profesores tienen unas RS estables de las finalidades de la enseñanza de la historia. Estas están influenciadas por las diferentes perspectivas teóricas de la historia presentes en la formación disciplinar. Asimismo, se puede señalar que el período de prácticas influye poco en su cambio.

\section{Discusión de resultados}

La formación inicial del profesorado de historia tiene como propósito desarrollar las competencias necesarias para que estos se desempeñen de manera adecuada en su actividad profesional. En este proceso se intenta proporcionar conocimientos y experiencias que influyan en cómo los futuros 
profesores representan la enseñanza. Esta influencia es diferencial, tal como lo muestran los resultados. Por un lado, está el predominio del componente disciplinar y, por otro, en menor medida, las experiencias de las prácticas iniciales, coincidiendo con lo planteado por María Alejandra CoudannesAguirre (2010). Llegar a esta identificación es un elemento diferenciador de los trabajos realizados por Susan Adler y Jesse Goodman (1983) y Joan Pagès-Blanch (1999), porque hicieron aproximaciones de carácter general, mientras que el presente estudio logró identificar y caracterizar en buena medida el contenido y la organización de la representación a través de las tipologías.

El análisis muestra que las RS que construye el profesorado durante la formación son sólidas y estables. Esto coincide con los hallazgos de Richard Harris (2012), quien en su estudio mostró la poca influencia de las prácticas en la manera de entender la enseñanza de la historia. Ante esta situación, ¿qué factores pueden propiciar un cambio significativo en las RS sobre la enseñanza de la historia?, ¿de qué manera en la formación inicial se pueden propiciar las condiciones para que los futuros profesores revisen sus RS? Estas preguntas motivan el debate académico y la realización de investigaciones que contribuyan a responderlas.

El peso de la perspectiva crítica en las RS acerca de la enseñanza de la historia coincide con los hallazgos de Susan Adler y Jesse Goodman (1983), Arja Virta (2009) y Michiel Voet y Bram de Wever (2016), pero se aleja de lo encontrado por Kaya Yilmaz (2008). Las coincidencias van en la línea de representar la enseñanza de la historia orientada a movilizar para la transformación social. Los hallazgos de Miguel Ángel Jara (2012) fueron similares y señalan que el profesorado en formación intenta asumir la perspectiva crítica, pero esto no implica que eso se refleje en sus prácticas de enseñanza. En contraste, Kaya Yilmaz (2008) encontró que los futuros profesores tendían a pensar la enseñanza de la historia como algo objetivo, que para efectos del presente estudio corresponde a las dos primeras tipologías, es decir, las minoritarias.

La configuración de las tipologías muestra la existencia de unas RS heterogéneas, lo que evidencia la influencia de diferentes escuelas historiográficas y la manera de entender la enseñanza de la historia. Al analizar el peso de las tipologías, se puede señalar que los planteamientos cercanos a la Nueva Historia, la Escuela de los Annales y la Historia Social tienen mayor reconocimiento y apropiación por parte de los profesores en formación. La influencia de las diferentes escuelas historiográficas muestra el debate amplio sobre el tipo de historia que se debe enseñar en la educación obligatoria (Arias-Gómez, 2015; Sánchez-Vásquez, 2013). 
El proceso seguido en la investigación revela que la identificación de las RS acerca de la enseñanza de la historia es un punto de partida para generar procesos de reflexión sobre el profesorado en formación (Gutiérrez \& Pagès-Blanch, 2014), que los lleven a pensar en cómo conciben su enseñanza (González-Valencia \& Santisteban-Fernández, 2016; Jara, 2012; Pagès-Blanch, 1999; Virta, 2002; Voet \& De Wever, 2016). En estos es necesario considerar los objetivos (González-Valencia, 2013; Harris, 2012; Wineburg, 2001), la selección de conceptos, la construcción de propuestas metodológicas y la evaluación. En otras palabras, el trabajo con las RS puede ser uno de los ejes de la formación en didáctica de la historia, tal como han sugerido Susan Adler y Jesse Goodman (1983), Todd Dinkelman (1989) y Joan Pagès-Blanch (1999) en los trabajos pioneros en la línea de investigación en la que se inscribe el presente estudio.

Un aspecto relevante en el conjunto de respuestas de los estudiantes es la ausencia de referencias a la enseñanza del conflicto, la recuperación de la memoria o la enseñanza de temas controversiales o cuestiones socialmente vivas. Lo anterior difiere de los hallazgos de María Isabel ToledoJofré, Abraham Magendzo-Kolstrein, Virna Gutiérrez-Gianella y Ricardo Iglesias-Segura (2015) en su estudio en Chile, quienes sí encontraron algunas referencias respecto a la vulneración de los derechos humanos. Esta ausencia llama la atención, dado el contexto sociopolítico que atraviesa el país, donde la enseñanza de la historia debe tener un lugar relevante para promover en los estudiantes la comprensión de lo que ha sucedido durante los últimos 60 años, para el desarrollo de su conciencia y de su pensamiento histórico. Esta ausencia sugiere la necesidad de continuar investigando sobre la manera en que el futuro profesorado entiende el conflicto y la enseñanza de este.

\section{Conclusiones}

Los resultados muestran que las RS de los futuros profesores acerca de las finalidades de la enseñanza de la historia son diversas y están influenciadas por la formación que reciben en la universidad, concentrada en el componente disciplinar y en las diferentes escuelas de pensamiento histórico. El peso del componente disciplinar configura el núcleo de la representación y en cada una de las tipologías asume unas características diferentes. En conexión con lo anterior, las RS tienden a centrarse en la perspectiva crítica o emancipatoria (Ross, 2006).

De igual manera, se resalta que la experiencia de las prácticas de fin de carrera tiene poca influencia en el cambio de las RS y las muestra como estables, como lo evidencian los resultados de los dos cuestionarios. 
Es necesario señalar que en una investigación que trabaja con las categorías de las RS, los datos estadísticos muestran un panorama general y, por lo tanto, sería pertinente un estudio cualitativo longitudinal a lo largo del proceso formativo para tener una comprensión más amplia al respecto.

Llama la atención la ausencia de referencias a la formación en didáctica de la historia, lo cual reafirma que, para los participantes del estudio, el conocimiento de la historia ocupa un lugar central y el conocimiento didáctico para su enseñanza está en un segundo plano. Los participantes hacen mención a conceptos de primer y segundo orden; sin embargo, llamó la atención la ausencia de referencias a los procesos psicológicos relacionados con habilidades de pensamiento histórico.

En términos metodológicos, la construcción de las tipologías fue una alternativa que permitió comprender con mayor profundidad las RS que construyen los futuros profesores. Esta elaboración fue un proceso que posibilitó acceder a matices que tienden a quedar ocultos en los análisis cuantitativos. Esto muestra la pertinencia de los enfoques mixtos en la investigación en didáctica de la historia.

Los hallazgos sugieren la importancia de avanzar hacia investigaciones longitudinales que permitan comprender lo que sucede durante la formación, la experiencia de las prácticas y los primeros años de actividad profesional. Este tipo de trabajos permitiría conocer con mayor profundidad la contribución a las RS de los diferentes componentes de la formación — disciplinares, didácticos, pedagógicos, las prácticas-, así como los cambios que puedan darse en el tiempo.

\section{Sobre los autores}

Gustavo Alonso González-Valencia es doctor en Didáctica de las Ciencias Sociales, Universidad Autónoma de Barcelona, UAB, España. Profesor Serra Húnter del Departamento de Didáctica de la Lengua, la Literatura y las Ciencias Sociales de la misma universidad. Miembro del Grupo de Investigación en Didáctica de las Ciencias Sociales (GREDICS), Universidad Autónoma de Barcelona, UAB, España.

Antoni Santisteban-Fernández es doctor en Didáctica de las Ciencias Sociales, Universidad Autónoma de Barcelona, UAB, España. Profesor titular, Departamento de Didáctica de la Lengua y la Literatura, y de las Ciencias Sociales, Universidad Autónoma de Barcelona, UAB, España. Miembro del Grupo de Investigación en Didáctica de las Ciencias Sociales (GREDICS), Universidad Autónoma de Barcelona, UAB, España.

Joan Pagès-Blanch es doctor en Ciencias de la Educación, Universidad de Barcelona, UAB, España. Profesor catedrático, Departamento de Didáctica de la 
Lengua y la Literatura, y de las Ciencias Sociales. Miembro del Grupo de Investigación en Didáctica de las Ciencias Sociales (GREDICS), Universidad Autónoma de Barcelona, UAB, España.

\section{Referencias}

Adler, S. \& Goodman, J. (1983). What is Social Studies? Student Teacher Perspectives. Presentation to the National Council for the Social Studies. San Francisco, California. Disponible en: http://files.eric.ed.gov/fulltext/ED240033.pdf

Arias Gómez, D. (2015). La enseñanza de las ciencias sociales en Colombia: lugar de las disciplinas y disputa por la hegemonía de un saber. Revista de Estudios Sociales, 52, 134-146. Disponible en: http://www.scielo.org.co/pdf/res/ n52/n52a10.pdf

Bardin, L. (1986). El análisis de contenido. Madrid: Ediciones AKAL.

Benejam-Argimbau, P. (2002). Las finalidades de la educación social. En P. Benejam Argimbau \& J. Pagès Blanch (eds.). Enseñar y aprender ciencias sociales, geografía e historia en la educación secundaria, 33-52. Barcelona: Horsori.

Catling, S. (2004). An Understanding of Geography: The Perspectives of English Primary Trainee Teachers. GeoJournal, 60 (2), 149-158. DOI: 10.1023/B:GEJO.0000033575.54510.c6

Colombia (1994). Ley 115 de 1994, por la cual se expide la ley general de educación. Diario Oficial, 41.214, 8 de febrero de 1994. Disponible en: http:// www.mineducacion.gov.co/1621/articles-85906_archivo_pdf

Colombia, Ministerio de Educación Nacional, MEN (2004). Resolución 1036, 22 de abril de 2004. Disponible en: http://www.mineducacion.gov.co/1621/ article-86386.html

Coudannes-Aguirre, M. A. (2010). La formación del profesor de historia en la universidad argentina. La creciente distancia entre investigación/docencia y teoría/práctica. Antíteses, 3 (6), 975-990. Disponible en: www.uel.br/re vistas/uel/index.php/antiteses/article/download/4579/7056

Davini, M. C. (ed.) (2002). De aprendices a maestros: enseñar y aprender a enseñar. Buenos Aires: Educación Papers Editores.

Dewey, J. (1978). Democracia y Educación: una introducción a la filosofía de la educación. $8^{\mathrm{a}}$ ed. Buenos Aires: Losada.

Dijk, T. van (1992). La ciencia del texto: un enfoque interdisciplinario. Barcelona: Paidós.

Dinkelman, T. (1989). Conceptions of Democratic Citizenship in Preservice Social Studies Teacher Education: A Case Study. Quebec: American Educational Research Association. Disponible en: https://files.eric.ed.gov/fulltext/ ED444970.pdf

Evans, R. (1991). Concepciones del maestro de historia. Boletín de Didáctica de las Ciencias Sociales, 3 y 4, 61-94. Disponible en: http://www.didacticaciencias-sociales.org/boletines_archivos/3_4-1991.pdf

Flick, U. (2007). Introducción a la investigación cualitativa. $2^{\mathrm{a}}$ ed. Madrid: Ediciones Morata.

Gimeno, P. (1999). La teoría crítica de Habermas y la educación: hacia una didáctica crítico-comunicativa. Con-ciencia Social: Anuario de Didáctica de 
la Geografía, la Historia y las Ciencias Sociales, (3), 13-41. Disponible en: https://dialnet.unirioja.es/ejemplar/94327

Gómez-Carrasco, J.; Ortuño-Molina, J. \& Molina-Puche, S. (2014). Aprender a pensar históricamente. Retos para la historia en el siglo XXI. Revista Tempo e Argumento, 6 (11), 5-27. Disponible en: http://periodicos.udesc.br/index. php/tempo/article/viewFile/2175180306112014005/3052

Gómez-Esteban, J. H. (2004). La construcción del conocimiento social en la escuela. $2^{a}$ ed. Bogotá: Sección Publicaciones Universidad Distrital Francisco José de Caldas.

González-Valencia, G. A. (2013). El profesorado en formación y las finalidades de la enseñanza de las ciencias sociales. Uni-pluri/versidad, 13 (2), 24-34. Disponible en: https://aprendeenlinea.udea.edu.co/revistas/index.php/unip/ article/download/16971/14695, https://aprendeenlinea.udea.edu.co/revis tas/index.php/unip/issue/view/1593

González-Valencia, G. A. \& Santisteban-Fernández, A. (2014). Una mirada a la investigación en didáctica de las ciencias sociales. Revista Latinoamericana de Estudios Educativos (Colombia), 10 (1), 7-17. Disponible en: https://www. redalyc.org/articulo.oa?id=134139791001

González-Valencia, G. A. \& Santisteban-Fernández, A. (2016). La formación ciudadana en la educación obligatoria en Colombia: entre la tradición y la transformación. Educación y Educadores, 19 (1), 89-102. DOI: 10.5294/ edu.2016.19.1.5. Disponible en: http://educacionyeducadores.unisabana. edu.co/index.php/eye/article/view/5533/4210

Gutiérrez, M. C. \& Pagès-Blanch, J. (2014). La formación del profesorado en didáctica de las Ciencias Sociales: aportaciones interdisciplinares. En J. Pagès-Blanch \& A. Santisteban-Fernández (eds.). Una mirada al pasado y un proyecto de futuro: investigación e innovación en didáctica de las ciencias sociales, vol. 2, 261-284. Barcelona: Universitat Autònoma de Barcelona, Servei de Publicacions, Asociación Universitaria de Profesores de Didáctica de las Ciencias Sociales. Disponible en: http://didactica-ciencias-sociales.org/ wp-content/uploads/2013/07/XXVSIMPO2.pdf

Habermas, J. (1966). Teoría y Praxis: Ensayos de Filosofía Social. Buenos Aires: Sur. Harris, R. (2012). 'Purpose' as a Way of Helping White Trainee History Teachers Engage with Diversity Issues. Education Sciences, 2 (4), 218-241. doi: https:// doi.org/10.3390/educsci2040218. Disponible en: https://core.ac.uk/down load/pdf/1442835.pdf

Henríquez, R. \& Pagès-Blanch, J. (2004). La investigación en didáctica de la historia. Educación XX1: Revista de La Facultad de Educación, 7, 63-84. Disponible en http://revistas.uned.es/index.php/educacionXX1/article/view/330/286

Hernández-Cervantes, L. (2014). ¿Quiénes enseñan el medio social en la educación infantil? Representaciones sociales sobre los modelos de profesora de educación infantil para la enseñanza de las ciencias sociales. Revista Latinoamericana de Estudios Educativos (Colombia), 10 (1), 113-135. Disponible en: http://vip.ucaldas.edu.co/latinoamericana/downloads/Latinoamerica na10(1)_6.pdf

Hernández-Sampieri, H.; Fernández-Collado, C. \& Baptista-Lucio, P. (2014). Metodología de la investigación. Ciudad de México: McGraw-Hill. Disponible en: 
https://www.esup.edu.pe/descargas/dep_investigacion/Metodologia\%20 de\%20la\%20investigaci\%C3\%B3n\%205ta\%20Edici\%C3\%B3n.pdf

Íñiguez-Rueda, L. (2006). Análisis del discurso: manual para las ciencias sociales. $3^{\mathrm{a}} \mathrm{ed}$. Barcelona: Editorial de la Universitat Oberta de Catalunya, UOC.

Jara, M. Á. (2012). Representaciones y enseñanza de la historia reciente-presente: Estudio de casos de estudiantes en formación inicial y en su primer año de docencia. Enseñanza de las Ciencias Sociales: Revista de Investigación, 11, 15-29. Disponible en: https://www.raco.cat/index.php/EnsenanzaCS/article/ view/263452/350946

Jara, M.; Salto, V. \& Értola, F. (2016). Representaciones y perspectivas prácticas sobre las finalidades de la enseñanza de la historia en los/las estudiantes del profesorado. Clío \& Asociados, La Historia Enseñada, 23, 69-78. Disponible en: https://www.clio.fahce.unlp.edu.ar/article/view/7898/8796

Jodelet, D. (1984). La representación social: fenómenos, conceptos y teoría. En S. Moscovici (ed.). Introducción a la Psicología Social, 469-493. Barcelona: Paidós.

Kitson, A.; Steward, C. \& Husbands, C. (2015). Didáctica de la historia en secundaria obligatoria y bachillerato. Madrid: Morata.

Liston, D. \& Zeichner, K. (2003). Formación del profesorado y condiciones sociales de la escolarización. Barcelona: Ediciones Morata.

Mora, M. (2002). La teoría de las representaciones sociales de Serge Moscovici. Athenea Digital. Revista de Pensamiento e Investigación Social, 1 (2), 1-25. DOI: https://doi.org/10.5565/rev/athenead/v1n2.55. Disponible en: https:// atheneadigital.net/article/view/n2-mora/55-pdf-es

Pagès-Blanch, J. (1999). Las representaciones previas de los estudiantes de maestro de Ciencias Sociales, Geografía e Historia. Teoría y Didáctica de las Ciencias Sociales, 4, 161-178. Disponible en: http://www.saber.ula.ve/bitstream/ handle/123456789/23928/num4_joan_pages.pdf;jsessionid=6289527546 8BF29B3C6DEFF7085BF185? sequence $=1$

Pagès-Blanch, J. (2002a). Aprender a enseñar historia y ciencias sociales: el currículo y la Didáctica de las Ciencias Sociales. Pensamiento Educativo, 30, 255-269. Disponible en: http://www.pensamientoeducativo.uc.cl/files/jour nals/2/articles/222/public/222-523-1-PB.pdf

Pagès-Blanch, J. (2002b). Líneas de investigación en didáctica de las ciencias sociales. En P. Benejam Arguimbau \& J. Pagès Blanch (eds.). Enseñar y aprender Ciencias Sociales, Geografía e Historia en la educación secundaria, 209-226. Barcelona: Horsori.

Pagès-Blanch, J. (2004). Enseñar a enseñar Historia: la formación didáctica de los futuros profesores. En E. Nicolás Marín \& J. A. Gómez Hernández (coords.). Miradas a la Historia: reflexiones historiográficas en recuerdo de Miguel Rodríguez Llopis, 155-178. Disponible en: http://libros.um.es/editum/catalog/ view/991/1621/1161-1, https://www.um.es/campusdigital/Libros/textoCom pleto/historia/12pages.pdf

Pagès-Blanch, J. (2007). ¿Qué se debería enseñar de historia hoy en la escuela obligatoria?, ¿qué deberían aprender, y cómo, los niños y las niñas y los y las jóvenes del pasado? Revista Digital Escuela de Historia, 1 (6), 17-30. Disponible en: https://www.redalyc.org/articulo.oa?id=63810603 
Pagès-Blanch, J. (2009). Competencia social y ciudadana. Aula de Innovación Educativa, 187, 7-11. Disponible en: https://competenciasaula.webnode. es/files/200000024-d8f69d9ee9/competencia\%20social\%20y\%20ciu dadana.pdf

Pagès-Blanch, J. \& Santisteban-Fernández, A. (2014). Una mirada desde el pasado al futuro en la didáctica de las Ciencias Sociales. En A. SantistebanFernández \& J. Pagès-Blanch (eds.). Una mirada al pasado y un proyecto de futuro: investigación e innovación en didáctica de las ciencias sociales, vol. 1, 17-41. Barcelona: Universitat Autònoma de Barcelona, Servei de Publicacions, Asociación Universitaria de Profesores de Didáctica de las Ciencias Sociales. Disponible en: http://didactica-ciencias-sociales.org/wp-content/ uploads/2013/11/XXVSIMPO1_v2.pdf

Pantoja-Suárez, P. T.; Loaiza-Zuluaga, Y. \& Posada-López, R. (2014). Ecos del ayer, el hoy y el mañana: la conciencia histórica como concepto y propuesta en la formación de licenciados en ciencias sociales. Revista Latinoamericana de Estudios Educativos, 10 (1), 136-157. Disponible en: http://vip.ucaldas.edu. co/latinoamericana/downloads/Latinoamericana10(1)_7.pdf

Phoenix, A.; Howarth, C. \& Philogène, G. (2017). The Everyday Politics of Identities and Social Representations: A Critical Approach. Papers on Social Representations, 26 (1). 2.1-2.21. Disponible en: http://psr.iscte-iul.pt/index. php/PSR/article/view/82

Pipkin, D. (2009). Pensar lo social. Buenos Aires, Argentina: La Crujía.

Pluckrose, H. (1996). Enseñanza y aprendizaje de la historia. Madrid: Ediciones Morata.

Prats, J. (1999). Líneas de investigación en didáctica de las ciencias sociales. História \& Ensino, Revista do Laboratório de Ensino de História/UEL, Universidade Estadual de Londrina. Brasil, 9, 133-155. DOI: http://dx.doi. org/10.5433/2238-3018.2003v9n0p133. Disponible en: http://www.uel. br/revistas/uel/index.php/histensino/article/view/12080/10618

Reitano, P. \& Green, N. (2013). Beginning Teachers' Conceptual Understandings of Effective History Teaching: Examining the Change from "Subject Knowers" to "Subject Teachers". Asia-Pacific Journal of Teacher Education, 41 (2), 197-217. http://doi.org/10.1080/1359866X.2013.777027

Rodríguez-Ávila, S. P. (2014). Enseñanza y aprendizaje de la historia en Colombia. 1990-2011. En S. Plá Pérez \& J. Pagès-Blanch (eds.). La investigación en la enseñanza de la historia en América Latina, 109-154. México: Universidad Pedagógica Nacional.

Rodríguez-Salazar, T. (2007). Sobre el estudio cualitativo de la estructura de las representaciones sociales. En T. Rodríguez-Salazar \& M. de L. García-Curiel (eds.). Representaciones sociales. Teoría e investigación, 157-190. Guadalajara, México: Universidad de Guadalajara, Centro Universitario de Ciencias Sociales y Humanidades, Editorial CUCSH-UdeG.

Ross, E. W. (2006). The Social Studies Curriculum. New York: State University of New York, SUNY Press.

Ruiz, J. (2008). Análisis sociológico del discurso: mínimo común denominador. En E. García-Álvarez \& J. López-Sintas (eds.). Actas de la Conferencia Iberoamericana de Investigación Cualitativa, IBERACUAL, vol. 1, 241-262. 
Barcelona: Universitat Autònoma de Barcelona, UAB, Centre d'Estudis i Recerca d'Humanitats, Universitat Oberta de Catalunya, UOC. Disponible en: https://www.dropbox.com/s/v4ytt|6noh2os1c/Actas_Iberacual_2008-vol-I. $\operatorname{pdf} ? \mathrm{~d}=0$

Sánchez-Agustí, M. (2011). Ciudadanía y enseñanza de la historia. Resultados de una intervención en la formación de maestros. Didáctica de las Ciencias Experimentales y Sociales, 25, 3-15. Disponible en: https://core.ac.uk/down load/pdf/71003689.pdf

Sánchez-Vásquez, N. (2013). Debates y discursos en torno a las ciencias sociales escolares entre 1984 y 2010 en Colombia. Uni-pluri/versidad, 13 (2), 69-80. Disponible en: http://tesis.udea.edu.co/bitstream/10495/8659/1/Sanchez Nubia_2013_DebatesCienciasSocialesEscolares.pdf

Santisteban-Fernández, A. (2009). Cómo trabajar en clase la competencia social y ciudadana. Aula de Innovación Educativa, 187, 12-15. Disponible en: https:// core.ac.uk/download/pdf/132092913.pdf

Santisteban-Fernández, A. (2010). La formación de competencias de pensamiento histórico. Clío \& Asociados: La Historia Enseñada, 14, 34-56. Disponible en: http://www.memoria.fahce.unlp.edu.ar/art_revistas/pr.4019/pr.4019.pdf

Santisteban-Fernández, A. (2017). Del tiempo histórico a la conciencia histórica: cambios en la enseñanza y el aprendizaje de la historia en los últimos 25 años. Diálogo Andino, 53, 87-99. https://doi.org/10.4067/S071926812017000200087. Disponible en: https://scielo.conicyt.cl/pdf/rda/ n53/0719-2681-rda-53-00087.pdf

Singer, A. J. (2008). Social Studies for Secondary Schools: Teaching to Learn, Learning to Teach. $3^{\text {rd }}$ ed. New York: Taylor \& Francis. https://doi.org/10. 4324/9780203891872

Strauss, A. \& Corbin, J. (2002). Bases de la investigación cualitativa: técnicas y procedimientos para desarrollar la teoría fundamentada. Medellín: Universidad de Antioquia. Disponible en: https://diversidadlocal.files.wordpress. com/2012/09/bases-investigacion-cualitativa.pdf

Teddlie, C. \& Tashakkori, A. (2009). Foundations of Mixed Methods Research: Integrating Quantitative and Qualitative Approaches in the Social and Behavioural Sciences. Los Angeles: SAGE Publications.

Toledo-Jofré, M. I.; Magendzo-Kolstrein, A.; Gutiérrez-Gianella, V. \& IglesiasSegura, R. (2015). Enseñanza de "temas controversiales" en la asignatura de historia y ciencias sociales desde la perspectiva de los profesores. Estudios Pedagógicos, 41 (1), 275-292. Disponible en: https://scielo.conicyt.cl/pdf/ estped/v41n1/art16.pdf

Valencia-Castañeda, L. (2014). Aprender a ser profesor y profesora de Historia. Los propósitos de la enseñanza en la formación del profesorado de Historia y Ciencias Sociales de la Universidad de Santiago de Chile. Tesis doctoral, Barcelona, Universitat Autònoma de Barcelona, UAB. Disponible en: https:// ddd.uab.cat/pub/tesis/2014/hdl_10803_284965/lvvc1de1.pdf

Valencia-Castañeda, L.; Pérez, A. \& Jara, L. (2014). El papel de los profesores guías en el desarrollo de una práctica reflexiva. En J. Pagès-Blanch \& A. Santisteban-Fernández (eds.). Una mirada al pasado y un proyecto de futuro: investigación e innovación en didáctica de las ciencias sociales, vol. 2, 
347-354. Barcelona: Universitat Autònoma de Barcelona, Servei de Publicacions, Asociación Universitaria de Profesores de Didáctica de las Ciencias Sociales. Disponible en: http://didactica-ciencias-sociales.org/wp-content/ uploads/2013/07/XXVSIMPO2.pdf

Villalón-Gálvez, G. (2012). Las representaciones sociales y las perspectivas prácticas sobre la ciudadanía de los profesores y estudiantes de profesorado en Historia, Geografía y Ciencias Sociales en Chile. En N. de AlbaFernández, F. F. García-Pérez \& A. Santisteban-Fernández (eds.). Educar para la participación ciudadana en la enseñanza de las Ciencias Sociales, vol. 1, 303-312. Sevilla: Asociación Universitaria de Profesores de Didáctica de las Ciencias Sociales. Disponible en: https://dialnet.unirioja.es/servlet/ articulo?codigo $=3978582$

Villalón-Gálvez, G. (2015). Creencias, propósitos y prácticas de la enseñanza de la historia: el caso de Mariana. Enseñanza de las Ciencias Sociales: Revista de Investigación, 14, 71-81. Disponible en: https://www.raco.cat/index.php/ EnsenanzaCS/article/view/303622/393320

Virta, A. (2002). Becoming a History Teacher: Observations on the Beliefs and Growth of Student Teachers. Teaching \& Teacher Education: An International Journal of Research and Studies, 18 (6), 687-698. Disponible en: http:// citeseerx.ist.psu.edu/viewdoc/download?doi=10.1.1.486.3595\&rep=rep1 \&type $=p d f$

Virta, A. (2009). Learning to Teach History in Culturally Diverse Classrooms. Intercultural Education, 20 (4), 285-297. https://doi.org/10.1080/1467598 0903351920

Voet, M. \& De Wever, B. (2016). History Teachers' Conceptions of Inquiry-Based Learning, Beliefs about the Nature of History, and their Relation to the Classroom Context. Teaching and Teacher Education, 55, 57-67. http://doi. org/10.1016/j.tate.2015.12.008

Wineburg, S. (2001). Historical Thinking and Other Unnatural Acts: Charting the Future of Teaching the Past. Philadelphia: Temple University Press.

Yilmaz, K. (2008). Social Studies Teachers' Conceptions of History: Calling on Historiography. The Journal of Educational Research, 101 (3), 158-176. http:// doi.org/10.3200/JOER.101.3.158-176 